\title{
The use of custom 3D printed stereotactic frames for laser interstitial thermal ablation: technical note
}

\author{
Nicholas J. Brandmeir, MD, James Mclnerney, MD, and Brad E. Zacharia, MD \\ Department of Neurosurgery, Penn State Health Milton S. Hershey Medical Center, Hershey, Pennsylvania
}

Over the last several years, laser interstitial thermotherapy (LITT) has gained wide acceptance for the treatment of a myriad of cranial lesions. A wide variety of techniques for placement of the laser fiber have been reported with a spectrum of perceived benefits and drawbacks. The authors present the first report of a customized 3D printed stereotactic frame for LITT.

Approximately 1 week prior to surgery, 3-4 skull fiducials were placed after each of 5 patients received a local anesthetic as an outpatient. Radiographs with these fiducials were then used to create a trajectory to the lesion that would be treated with LITT. After the plan was completed, software was used to render a customized frame. On the day of surgery, the frame was attached to the implanted skull fiducials and the LITT catheter was placed. This procedure was carried out in 5 consecutive patients. In 2 patients, a needle biopsy was also performed.

Intraoperative and postoperative imaging studies confirmed the accurate placement of the LITT catheter and the lesion created. Mean operating room time for all patients was 45 minutes but only 26 minutes when excluding the cases in which a biopsy was performed.

To the best of the authors' knowledge, this is the first report of the use of a specific system, the STarFix microTargeting system, for use with LITT and brain biopsy. This system offers several advantages including fast operating times, extensive preoperative planning, no need for cranial fixation, and no need for frame or fiducial placement on the day of surgery. The accuracy of the system combined with these advantages may make this a preferred stereotactic method for LITT, especially in centers where LITT is performed in a diagnostic MRI suite.

http://thejns.org/doi/abs/10.3171/2016.8.FOCUS16219

KEY WORDS LITT; laser interstitial thermotherapy; high-grade glioma; glioblastoma; frameless; laser ablation

$\mathrm{O}$ VER the last few years, laser interstitial thermotherapy (LITT) has gained wide acceptance for the treatment of a myriad of neoplastic and nonneoplastic cranial pathologies. ${ }^{7}$ Numerous stereotactic techniques are available for accurate placement of LITT catheters including robot-assisted, ${ }^{2}$ optical frameless systems, ${ }^{1}$ MRI-assisted, ${ }^{3}$ and traditional frames. ${ }^{8}$ While all of these systems offer accuracy and versatility, they all have the drawbacks of requiring the patient to be fixed in head pins, to undergo fiducial scanning and trajectory planning on the day of the operation. These limitations may negatively impact operating room efficiency and expose patients to longer operative times and unnecessary risk.

We describe the use of a customized 3D printed frame system (STarFix microTargeting Platform, FHC Inc.). This system has been used successfully in other stereotactic applications with well-documented accuracy, ${ }^{4,9}$ In a small series of patients, we demonstrate that the STarFix system allows both the accurate placement of LITT catheters and the versatility of other systems while at the same time significantly shortening operative time and simplifying the operative day for the patient and surgeon. We review the workflow and technical nuances for completion of this procedure.

\section{Methods \\ Patients}

In the present study we included 5 consecutive patients in whom the NeuroBlate LITT system (Monteris Medical) 
in conjunction with the STarFix system was used over a 4-month period. All surgeries were identical with the exception that in 2 of the patients a biopsy was performed at the time of surgery. In one of these cases the biopsy failed to demonstrate neoplastic tissue on the frozen section, and the LITT procedure was aborted (Table 1).

\section{Surgical Technique}

After informed consent for the procedure was signed, small bone fiducial screws were implanted under sterile conditions after a minimal (approximately $1 \mathrm{~cm}$ ) scalp shave and administration of a local anesthetic. This was carried out either in the outpatient clinic or bedside in the hospital room on the final day of the patient's hospital admission. No sedation was necessary for these procedures, and all patients tolerated this portion of the procedure well. Fiducial placement was chosen by noting an approximation of the entry site on the patient's scalp and maintaining a minimum 3-cm distance from that site. After each fiducial site had been marked, shaved, and prepared, a No. 11 blade scalpel was used to make a small stab incision down to the skull. The tip of the knife was used to elevate the periosteum, and then a power driver (OsteoMed, Addison) was used to implant the titanium WayPoint fiducials (FHC Inc.). Three or four fiducials were used for a single trajectory, depending on the case and expected trajectory characteristics. After the fiducials were in place, a single 3-0 Monocryl suture was used to close each stab incision, and incisions were dressed with antibiotic ointment. A finecut CT scan was obtained with the fiducials for planning purposes, and the patient was then cleared for discharge from the clinic or hospital. We do not routinely provide systemic antibiotic prophylaxis for these patients either prior to fiducial implantation or on discharge.

Several factors influence the number of fiducials ( 3 vs 4) and the placement of fiducials. If the surgical trajectory is easy to approximate on the patient's scalp, the surgeon can feel confident in placing 3 fiducials roughly equidistant from the entry site. This will provide a stable frame and necessitate a minimal area of scalp to be accessed during the surgery and potentially aid operative positioning. On the other hand, if the proposed trajectory is difficult to approximate, then 4 fiducials offer the advantage of greater frame flexibility during planning and printing. For instance, if the fiducials turn out to be surrounding the trajectory, a 4-limbed frame can be printed. If a fiducial appears to be too close to or far from the proposed entry site, a 3-legged frame can be created and the superfluous fiducial removed at the time of the operation.

After implantation of the fiducials and acquisition of the fiducial scan, planning was accomplished using the microTargeting software on a desktop workstation. The fiducial CT scan was typically fused with a contrast-enhanced fine-cut MR image for target delineation. After an appropriate trajectory for the lesion had been planned, a 3D customized frame was rendered and shown with the patient imaging for approval (Fig. 1). After this plan and rendered frame had been verified as accurate, a production file was transferred over the Internet to the manufacturer.

Some considerations aid in the production of an optimal frame. It is possible that the frame as rendered will appear to have very thin legs or to encroach on the entry
TABLE 1. Patient characteristics

\begin{tabular}{ccccccc}
\hline $\begin{array}{c}\text { Patient } \\
\text { No. }\end{array}$ & $\begin{array}{c}\text { Age } \\
\text { (yrs) }\end{array}$ & Sex & \multicolumn{1}{c}{ Diagnosis } & Biopsy & LITT & $\begin{array}{c}\text { OR Time } \\
\text { (mins) }\end{array}$ \\
\hline 1 & 49 & M & High-grade glioma & No & Yes & 38 \\
\hline 2 & 78 & M & Glioblastoma & Yes & No & 78 \\
\hline 3 & 67 & M & Lung adenocarcinoma & No & Yes & 25 \\
\hline 4 & 71 & M & Lung adenocarcinoma & No & Yes & 16 \\
\hline 5 & 61 & M & Melanoma & Yes & Yes & 68 \\
\hline
\end{tabular}

$\mathrm{OR}=$ operating room.

site. The engineers at the manufacturer can be contacted to alter the automatically rendered frame to ensure the frame is stable and does not interfere with the trajectory. Also, because a cranial bolt must be placed through the frame, the frame must be created with sufficient height to accommodate this. This is often $1-$ to $1.5-\mathrm{cm}$ taller than would be used for a deep brain stimulator frame. Finally, although the patients in this series were all treated with the NeuroBlate system, the STarFix system is equally capable of accurately placing a Visualase catheter (Medtronic Inc.) as well.

The specification of the particular LITT system must be taken into account when planning a procedure. The NeuroBlate system has a dedicated set of appropriately sized reducing cannulas that interface with the STarFix system for their catheters that must be identified and available at the time of surgery. This is also the case for the Visualase system. Furthermore, if a biopsy is planned, the diameter of the biopsy needle is often different from the common LITT catheter diameters, and a reducing cannula will be necessary for this procedure as well. Most diameters of reducing cannulas are readily available, but if not, a specialized reducing cannula with appropriate specifications can be easily prototyped and delivered with the 3D printed frame in time for the case. The customizability and individualized nature of this stereotactic system confer on it some advantages in this regard when potentially dealing with multiple calibers of needle/catheter in the same case.

Approximately 1 week after the implantation of the fiducials, the patients returned to the hospital electively for the LITT procedure. The procedures were carried out after induction of general anesthesia while patients lay on litters in the operating room. The procedures do not require fixation of the head to the operating table, but NeuroBlate cranial fixation can be used if preferred. After the patient undergoes preparation and draping, the surgeon used scissors to expose the previously implanted fiducial screws, and polyetheretherketone (PEEK) standoff screws were inserted into the bone fiducials. After this step, the customized frame was attached to the stand-off screws (Fig. 2).

When the frame was in place, adapters were used to guide the appropriately sized power drill to the entry site, and the entry site was marked. It was then infiltrated with a local anesthetic, and a stab incision was made with a No. 11 blade scalpel. The power drill was then used to make a small twist-drill hole, under precise stereotactic guidance, through the frame. After the hole was created through the cranium, the dura was opened sharply with a trocar.

After the dura was opened, the metallic cranial bolt 

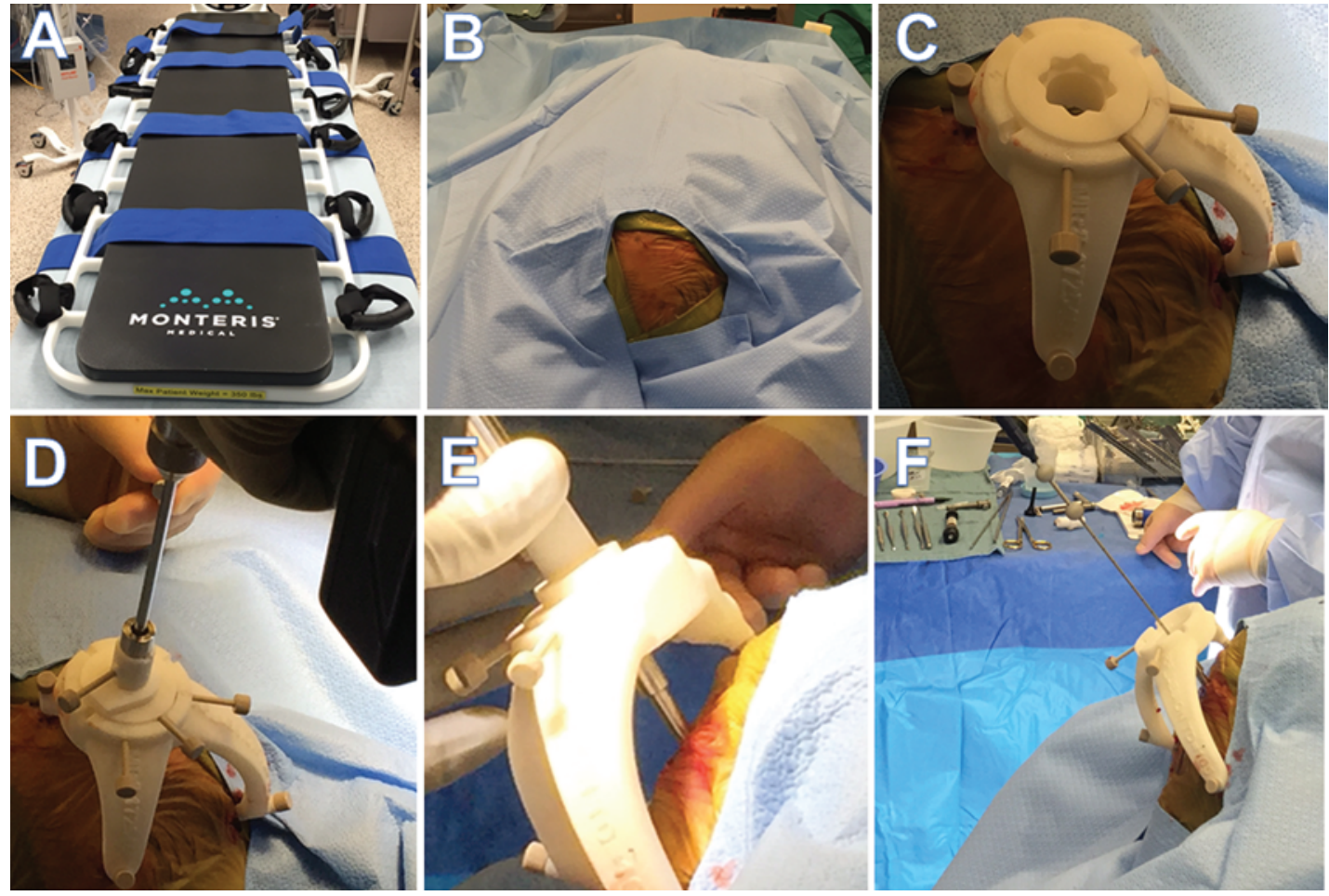

FIG. 1. Patient 2. Positioning of patient for surgery. A: Litter with stretcher (a pinning head clamp is part of the Monteris stretcher assembly, but is not necessary for patient positioning or accurate targeting). B: Positioning of the patient on the litter. C: Attachment of the customized frame with NeuroBlate adapter. D: Drilling of the hole with orthopedic drill. E: Placement of the targeting bolt with screwdriver still under stereotactic guidance. F: Needle biopsy through the frame and bolt.

for targeting the LITT system was inserted into the hole using a screwdriver provided by the LITT manufacturer, again under the stereotactic guidance of the frame. When the bolt was in place, the frame could be removed from the stand-off screws. At this point, final depth calculations were repeated based on the frame and LITT bolt and compared with the depth measured from preoperative planning and imaging. After the depth calculations were confirmed, the intracranial portion of the procedure was begun. This calculation accuracy depends on the distance from the STarFix platform to the top of the cranial bolt.

If a biopsy was to be undertaken, the biopsy needle (Medtronic Inc.) was introduced into the LITT bolt with a reducing cannula attached to the bolt of an appropriate diameter. After the needle reached the appropriate depth, core biopsy samples were taken in the standard fashion and sent either directly for permanent or for frozen analysis.

Several factors must be considered when preparing for a biopsy with this system. First and foremost, the outer diameter of the biopsy needle should match the inner diameter of either available STarFix adapters (such as those used for deep brain stimulation) or the inner diameter of the cranial bolt used for LITT. Also, it is important to note that the optimal depth for a biopsy will often be somewhat shallower than for the optimal LITT placement, and this must be accounted for prior to passing the needle. In general, most stereotactic needles are compatible with default STarFix adapter system.

After the biopsies were completed, a sterile cap was placed on the bolt. At this point, the operating room por- tion of the procedure was complete, and the patient was transferred to the diagnostic MRI suite.

In the MRI suite, the patient was first carefully positioned in the bore of the magnet, the LITT catheter was inserted to the appropriate depth under sterile conditions, and the NeuroBlate system was assembled. The LITT procedure was carried out in the standard fashion. ${ }^{6} \mathrm{Al}-$ though the WayPoint fiducials are titanium, they produce very little MRI artifact and no artifact near the lesions for ablation. Furthermore, these fiducials have been used successfully in other MRI-dependent procedures with success. ${ }^{1}$ Surgeons should be sure to place the fiducials at least several centimeters from the site of ablation to avoid any potential MRI artifact from the WayPoint anchors, but the nature of frame construction and routine anchor/fiducial placement should make this a largely moot point in practice. This is especially true since ablation targets are usually sufficiently deep to make almost any placement acceptable.

After the procedure was completed, the LITT catheter, bolt, stand-off, and fiducial screws were all removed. Each wound was closed with a single Monocryl stitch and dressed with antibiotic ointment. The patients were extubated and recovered in the postanesthesia care unit. Patients were observed overnight in the intermediate care unit, and then discharged to home on hospital Day 1 or 2 .

\section{Case Summary}

Patient characteristics are detailed in Table 1 . The mean 


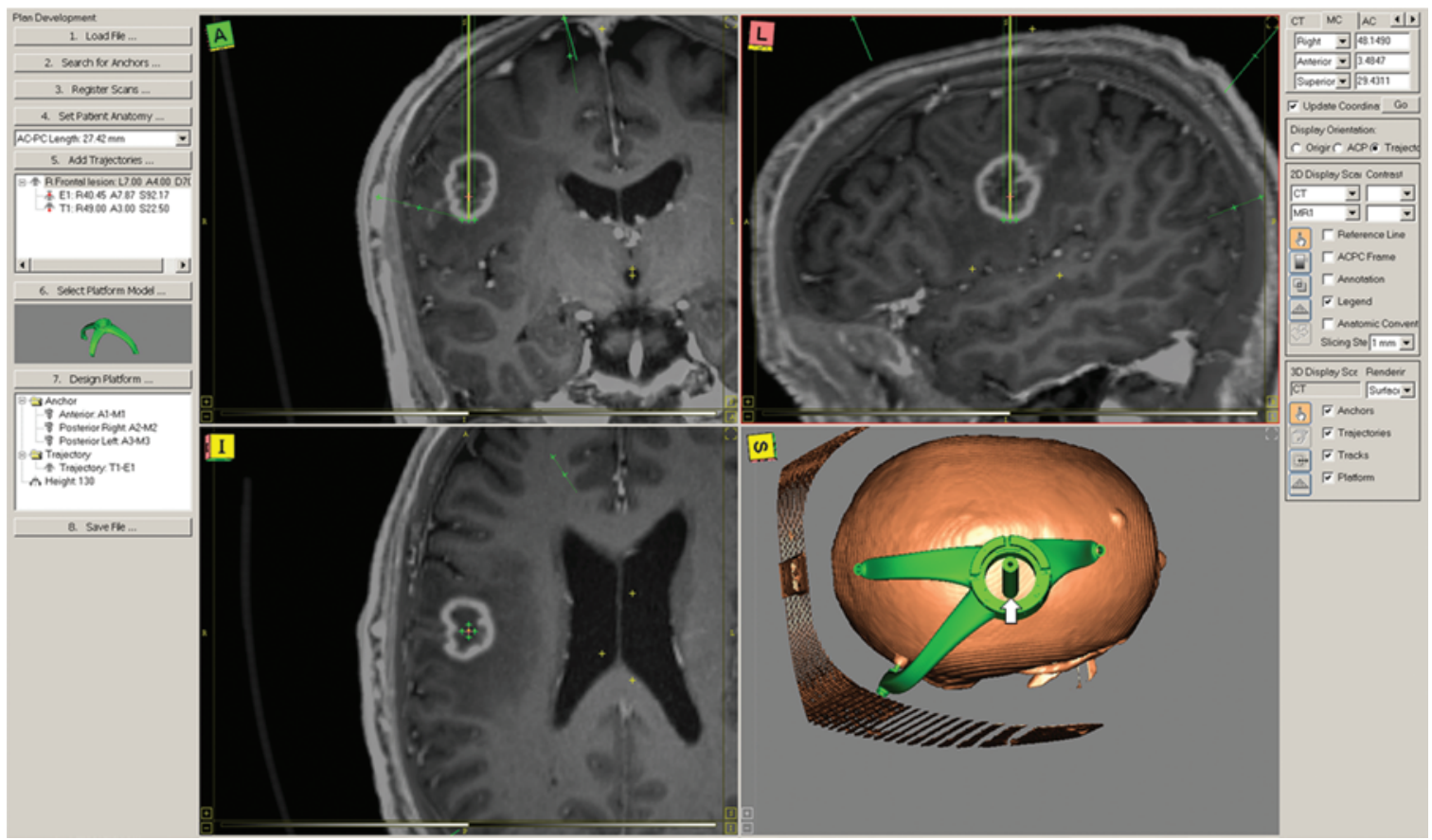

FIG. 2. Patient 3. Planning software and frame render. Images are T1-weighted postcontrast MR images merged with noncontrast high-definition CT scans. Views are trajectory views aligned with the catheter trajectory. The plug in the center of the frame render (arrow) is not part of the frame but, rather, a virtual tool to allow the surgeon to assess the height of the frame and ensure that it allows enough clearance for the cranial bolt.

operating room time was 45 minutes, but when biopsies were excluded, the mean time dropped to 26 minutes. The placement of the catheter was accurate in all cases, as confirmed by either intraoperative or postoperative imaging, and there were no procedure-related complications. In all 4 cases in which the LITT procedure was performed, the ablation was successful in destroying the total targeted area without causing injury to nearby structures (Fig. 3).

\section{Discussion}

To the best of our knowledge, this is the first report of the using the STarFix microTargeting platform for LITT as well as for needle biopsy. The system proved to be well tolerated by patients and to provide accuracy equivalent to other stereotactic systems currently available while causing no apparent morbidity. The STarFix system offers several important advantages over other systems as well. Because planning can be accomplished days ahead of time, there are no undue time pressures on the surgeon for planning, and this itself has the potential advantage of improving accuracy and reducing error. Also, because the customized frame is locked to the ideal trajectory planned by the surgeon, it allows tighter adherence to this optimal path, potentially increasing accuracy and decreasing morbidity.

Furthermore, because the system does not require head fixation, it can potentially decrease any morbidity associated with skull pins and/or frame placement and allow the procedure to be carried out on a litter rather than an operating table. This has the significant benefits of decreasing time in the operating room, improving surgery start times, and lowering morbidity associated with transport to and from the table. Without rigid skull fixation, anesthesia demands are reduced during the procedure, making the vital signs of the patient easier to control as well. Finally, without skull fixation the surgeon is free to make minor head positioning adjustments intraoperatively as necessary that would not be possible when using other systems without altering the trajectory or stereotactic accuracy. This last point is minor but can be helpful when implanting oblique or posterior trajectories.

This system does require skull fiducials, but this is not unique, and the other stereotactic systems currently in use for LITT often utilize a skull fiducial system. We believe, as do others, that better trajectory planning is achieved with skull fiducials when than with surface matching alone. ${ }^{5}$ The STarFix system offers the benefit of fiducial placement prior to the operative day in a location more comfortable for the patient and the surgeon (such as the outpatient clinic). The procedure does not cause significant pain and is well tolerated by patients. What we perceive as a benefit, however, can certainly be seen as a drawback. The procedure requires additional planning and logistical considerations. Our center under the direction of our coauthor Dr. McInerney has performed hundreds of lead placement procedures for deep brain stimulation. Thus, the protocol for fiducial placement was well entrenched at our center, rendering this slight modification rather simple. An additional drawback of the system is that it requires patients to have their skull fiducials in place for several days while at home. This can be irritating and mildly painful, but the pain is usually well managed with over-the-counter analgesics like acetaminophen and ibuprofen. Finally, the very short operating times and limited delays in the preoperative area, as well as for intraopera- 

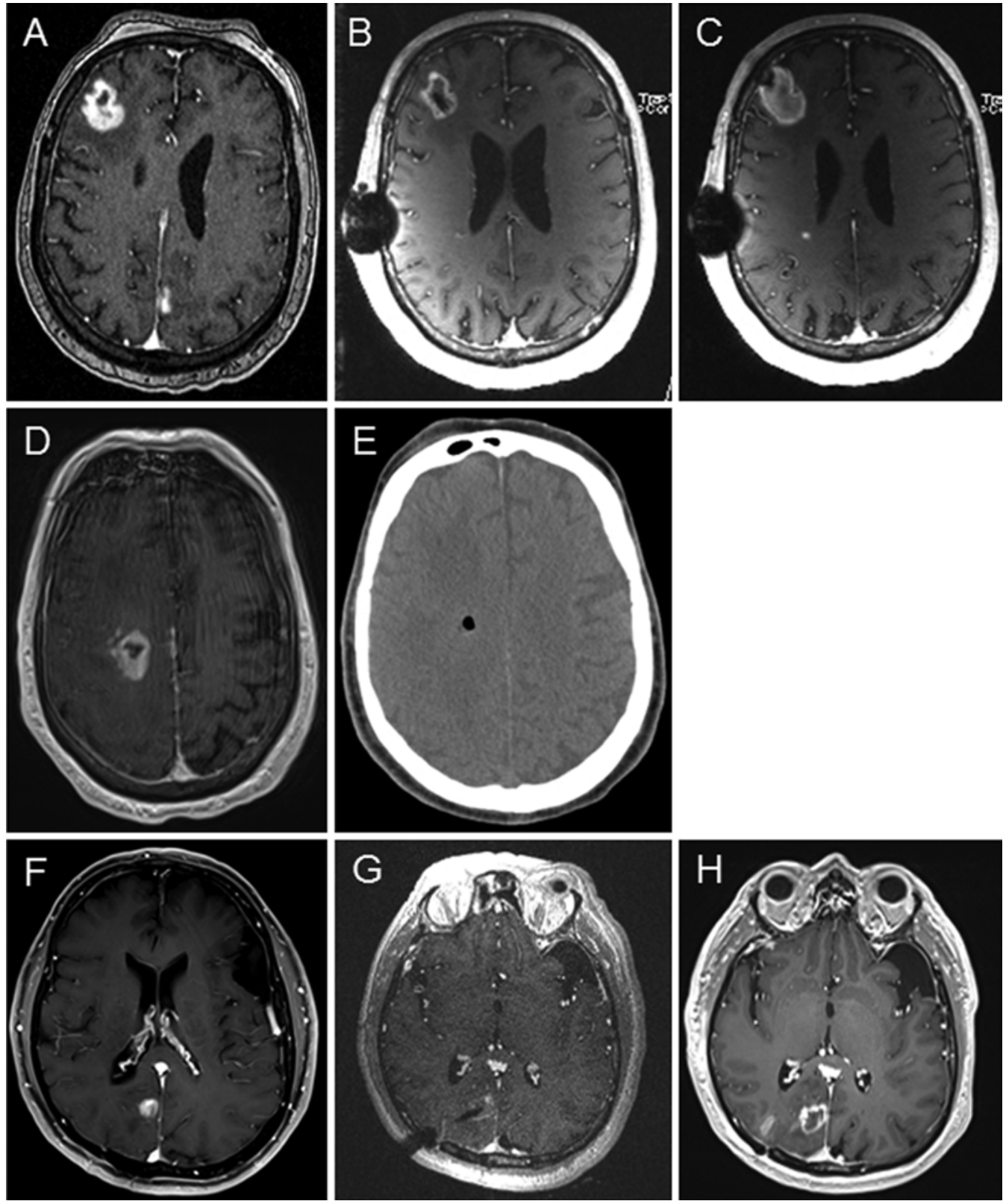

FIG. 3. Postcontrast T1-weighted MR images and CT scan demonstrating accuracy of the STarFix system. A: Preoperative image of Patient 4. B: Intraoperative image of Patient 4 demonstrating the NeuroBlate catheter in the lesion. C: Postoperative image of Patient 4 demonstrating accurate lesion placement. D: Preoperative image of Patient 2. E: Postoperative noncontrasted CT scan of Patient 2 demonstrating biopsy-associated pneumocephalus in the lesion, confirming accuracy. F: Preoperative image of Patient 5. G: Intraoperative image of Patient 5 confirming NeuroBlate catheter placement in the lesion. $\mathrm{H}$ : Immediate postoperative image of patient 5 showing accurate lesion placement.

tive positioning and transport, mean the STarFix system offers very reliable and fast operating times. This is especially important at centers where the LITT procedure is performed in a diagnostic MRI suite, as operating room and MRI efficiency is critical.

\section{Conclusions}

The STarFix microTargeting system is a fast, reliable, and accurate system for LITT procedures. This system can be used to perform LITT procedures with similar accuracy and safety as other stereotactic systems while offering several advantages for patient and surgeon convenience.

\section{References}

1. Attaar SJ, Patel NV, Hargreaves E, Keller IA, Danish SF: Accuracy of laser placement with frameless stereotaxy in magnetic resonance-guided laser-induced thermal therapy. Neurosurgery 11:554-563, 2015 
2. Brandmeir N, Acharya V, Sather M: Robot assisted stereotactic laser ablation for a radiosurgery resistant hypothalamic hamartoma. Cureus 8:e581, 2016

3. Chabardes S, Isnard S, Castrioto A, Oddoux M, Fraix V, Carlucci L, et al: Surgical implantation of STN-DBS leads using intraoperative MRI guidance: technique, accuracy, and clinical benefit at 1-year follow-up. Acta Neurochir (Wien) 157:729-737, 2015

4. Fitzpatrick JM, Konrad PE, Nickele C, Cetinkaya E, Kao C: Accuracy of customized miniature stereotactic platforms. Stereotact Funct Neurosurg 83:25-31, 2005

5. Helm PA, Eckel TS: Accuracy of registration methods in frameless stereotaxis. Comput Aided Surg 3:51-56, 1998

6. Mohammadi AM, Schroeder JL: Laser interstitial thermal therapy in treatment of brain tumors-the NeuroBlate System. Expert Rev Med Devices 11:109-119, 2014

7. Rahmathulla G, Recinos PF, Kamian K, Mohammadi AM, Ahluwalia MS, Barnett GH: MRI-guided laser interstitial thermal therapy in neuro-oncology: a review of its current clinical applications. Oncology 87:67-82, 2014

8. Rolston JD, Chang EF: Stereotactic laser ablation for hypothalamic hamartoma. Neurosurg Clin N Am 27:59-67, 2016
9. Stuart RM, Goodman RR: Novel use of a custom stereotactic frame for placement of depth electrodes for epilepsy monitoring. Neurosurg Focus 25(3):E20, 2008

\section{Disclosures}

Dr. Zacharia is a consultant for Monteris.

\section{Author Contributions}

Conception and design: Brandmeir, Zacharia. Acquisition of data: Brandmeir, Zacharia. Analysis and interpretation of data: all authors. Drafting the article: Brandmeir, Zacharia. Critically revising the article: all authors.

\section{Correspondence}

Nicholas J. Brandmeir, Department of Neurosurgery, Penn State Health Milton S. Hershey Medical Center, 30 Hope Dr., Hershey, PA 17011.email:nbrandmeir@hmc.psu.edu. 